\title{
Akutes Lungenversagen (ARDS)
}

\author{
Stefan Kluge und Annekatrin Sieweke
}

\section{Definition}

Das akute Lungenversagen, auch akutes respiratorisches Distress-Syndrom (ARDS, ,acute respiratory distress syndrome") genannt, wurde erstmalig 1967 durch Ashbaugh et al. beschrieben. Führendes Symptom ist ein akutes hypoxämisches Atemversagen im Verlauf einer pulmonalen (primäres ARDS) oder extrapulmonalen Erkrankung (sekundäres ARDS).

Nach der aktuellen Berlin-Definition der European Society of Intensive Care Medicine von 2012 liegt ein ARDS vor, wenn respiratorische Symptome innerhalb einer Woche nach bekanntem klinischen Ereignis neu auftreten oder sich verschlechtern. Zugleich sind in der radiologischen Bildgebung (Röntgen-Thorax oder CT-Thorax) bilaterale pulmonale Verschattungen nachzuweisen, die sich nicht ausschließlich durch Pleuraergüsse, Lungenkollaps oder Rundherde erklären lassen. Das respiratorische Versagen darf nicht vollständig auf ein Herzversagen oder eine Überwässerung zurückzuführen sein. Im Unterschied zu früheren Definitionen kann die kardiale

\section{S. Kluge $(\bowtie)$}

Klinik für Intensivmedizin, Universitätsklinikum Hamburg-Eppendorf, Hamburg, Deutschland E-Mail: s.kluge@uke.de

\section{A. Sieweke}

Institut für Intensivmedizin, Universitätsspital Zürich, Zürich, Schweiz

E-Mail: Annekatrin.Sieweke@usz.ch
Funktion durch eine Echokardiografie objektiviert werden.

Drei Schweregrade des ARDS werden durch das Ausmaß der Oxygenierungsstörung unterschieden. Maßgeblich ist der Oxygenierungsindex $\left(\mathrm{PaO}_{2} / \mathrm{FiO}_{2}\right)$ unter einem geringen positiven endexpiratorischen Beatmungsdruck (PEEP, „positive endexpiratory pressure“), der auch unter nichtinvasiver Beatmung (NIV, ,non invasive ventilation“) erhoben werden kann (Ranieri et al. 2012):

1. Mildes ARDS: $\mathrm{PaO}_{2} / \mathrm{FiO}_{2} 201-300 \mathrm{mmHg}$ unter PEEP $\geq 5 \mathrm{cmH}_{2} \mathrm{O}$

2. Moderates ARDS: $\mathrm{PaO}_{2} / \mathrm{FiO}_{2} 101-200 \mathrm{mmHg}$ unter PEEP $\geq 5 \mathrm{cmH}_{2} \mathrm{O}$

3. Schweres ARDS: $\mathrm{PaO}_{2} / \mathrm{FiO}_{2} \leq 100 \mathrm{mmHg}$ unter PEEP $\geq 5 \mathrm{cmH}_{2} \mathrm{O}$

\section{Pathophysiologie}

Pathophysiologische Grundlage des ARDS ist eine Schrankenstörung der alveolokapillären Einheit. Eine direkte pulmonale Schädigung über die Atemwege führt zu einer Verletzung des Alveolarepithels mit der Folge eines vermehrten Einstromes eiweißreicher Flüssigkeit in die Alveolen und einer verminderten Flüssigkeitsclearance aus dem Alveolarraum. Durch Schädigung der Pneumozyten Typ II ist die Surfactant-Bildung vermindert. Dies kann über den Verlust der alveolären Oberflächenspannung einen Kollaps der Alveolen verursachen. Bei extrapulmonaler Genese des ARDS 
wie Sepsis oder Schock entsteht das intraalveoläre Permeabilitätsödem durch Schädigung des pulmonalkapillären Endothels. Eine vermehrte Zytokin-induzierte Einwanderung neutrophiler Granulozyten in den Alveolarraum und in das Interstitium unterhält proinflammatorische Prozesse, die eine weitere Alveolarzellschädigung verursachen. Innerhalb von 72 Stunden setzen reparative Vorgänge ein mit Proliferation von Pneumozyten Typ II, Fibroblasten und Ablagerung neuer Matrix. Damit können 3 zeitlich überlappende und im individuellen Krankheitsverlauf unterschiedlich ausgeprägte Phasen unterschieden werden: 1 . eine exsudative oder inflammatorische Phase, 2. eine proliferative Phase und 3. eine fibrosierende Phase (Umbrello et al. 2017).

Eine weitere Folge der lokalen Inflammationsvorgänge ist eine Beeinträchtigung des Euler-Liljestrand-Reflexes. Dies führt zu einer fehlenden Vasokonstriktion in Lungenarealen mit einer verminderten alveolären Belüftung (Ventilation). Je nach Ausmaß entsteht ein relevantes Shuntvolumen, das mehr als $30 \%$ des Herzzeitvolumens betragen kann. Die Aktivierung der intravasalen Gerinnungskaskade kann zu Mikrothrombosierungen der pulmonalen Kapillaren führen. Es entstehen ventilierte, aber nicht durchblutete (perfundierte) Lungenareale, die zu einer Zunahme des Totraums führen. Unter dem ausgeprägten Permeabilitätsödem nimmt das Gewicht der Lunge bis um das Dreifache ihres Normalgewichts zu. Dies führt zu einer Abnahme der Lungencom- pliance. Eine Hypoxie-bedingte pulmonale Vasokonstriktion kann über einen erhöhten pulmonalarteriellen Druck eine Rechtsherzbelastung bis hin zu einem akuten Cor pulmonale verursachen. Entscheidend ist, dass ein ARDS zu einem inhomogenen Schädigungsmuster der Lunge führt, sodass neben belüfteten Arealen ödematöse, kollabierte und konsolidierte Lungenbereiche liegen.

\section{$3 \quad$ Epidemiologie}

Die Daten zur Inzidenz variieren in den einzelnen Studien erheblich und liegen zwischen 5,0 und 33,8 Fällen pro 100.000 Einwohner und Jahr. Neue prospektive Daten der Lung-Safe-Studie zeigen, dass 10,4 \% aller auf einer Intensivstation aufgenommenen Patienten ein ARDS haben, dies entspricht $23,4 \%$ aller invasiv beatmeten Patienten. Ein mildes ARDS liegt bei $30 \%$, ein moderates ARDS bei 46,6 \% und ein schweres ARDS bei $23,4 \%$ der Patienten vor. Die häufigsten Ursachen für ein ARDS sind Pneumonien (59,4\%), extrapulmonale Sepsis (16\%) und Aspiration (14\%) (Bellani et al. 2016).

Bei Fehlen der klassischen Risikofaktoren liegen in 7,5\% der Fälle hämatoonkologische Erkrankungen, Autoimmunerkrankungen, ein medikamentös verursachtes ARDS oder ein idiopathisches ARDS vor (Gibelin et al. 2016), siehe Tab. 1.

Tab. 1 Risikofaktoren für die Entstehung eines ARDS. (Nach: Ranieri et al. 2012; Gibelin et al. 2016)

\begin{tabular}{|c|c|c|}
\hline & Pulmonal & Extrapulmonal \\
\hline Häufig & $\begin{array}{l}\text { Pneumonie } \\
\text { Aspiration } \\
\text { Lungenkontusion } \\
\text { Inhalationstrauma } \\
\text { Pulmonale Vaskulitis } \\
\text { Ertrinkungsunfall }\end{array}$ & $\begin{array}{l}\text { Sepsis } \\
\text { Schweres Trauma } \\
\text { Pankreatitis } \\
\text { Schwere Verbrennungen } \\
\text { Nicht-kardiogener Schock } \\
\text { Medikamentenüberdosierung } \\
\text { TRALI (Transfusions-assoziiertes Lungenversagen) }\end{array}$ \\
\hline Selten & $\begin{array}{l}\text { Reperfusionsschaden } \\
\text { Fettembolie Syndrom }\end{array}$ & $\begin{array}{l}\text { Kardiopulmonaler Bypass } \\
\text { Malaria }\end{array}$ \\
\hline \multicolumn{3}{|c|}{$\begin{array}{l}\text { Ohne klassische Risikofaktoren: } \\
\text { - Autoimmunerkrankungen } \\
\text { - Hämatoonkologische Erkrankungen } \\
\text { - Medikamenten-induziert } \\
\text { - Idiopathisch }\end{array}$} \\
\hline
\end{tabular}




\section{$4 \quad$ Klinik}

Die klinischen Symptome eines ARDS sind durch die zugrunde liegende Erkrankung und das schwere hypoxämische Atemversagen charakterisiert: Dyspnoe, Tachypnoe, hoher Atemantrieb, Unruhe und ein Abfall der peripheren Sauerstoffsättigung trotz Steigerung der Sauerstoffzufuhr liegen in der Regel vor. Hinzukommen die Symptome der Grunderkrankung wie Fieber, Hypotonie, Tachykardie in der Sepsis oder paralytischer Ileus und abdominelles Kompartmentsyndrom bei Vorliegen einer Pankreatitis. Übergänge in ein Multiorganversagen mit akutem Cor pulmonale oder septischer Kardiomyopathie, septischer Enzephalopathie, Nieren- und Leberversagen sowie Rhabdomyolyse können im Verlauf auftreten und die Prognose einschränken. Wichtig ist eine genaue Beobachtung der Krankheitsdynamik, um bei einem schnellen therapierefraktären Atemversagen rechtzeitig Kontakt mit einem ARDS-Zentrum aufzunehmen und zeitnah die Indikation für eine extrakorporale Lungenunterstützung (ECMO, „extracorporeal membrane oxygenation") $\mathrm{zu}$ stellen (Kontakt und freie Aufnahmekapazitäten über www.ardsnetwork.de).

\section{$5 \quad$ Diagnostik}

Bei infektiöser Genese des ARDS wie Pneumonie oder extrapulmonaler Sepsis steht die Erregerdiagnostik (Trachealsekret/fiberoptische bronchoalveoläre Lavage [BAL], Blutkulturen, Urinkulturen und Legionellen-Antigennachweis im Urin) an erster Stelle. Eine erweiterte Erregerdiagnostik erfasst bei fehlender klinischer Besserung neben der Bakteriologie den Nachweis von Influenza, HSV (Herpex-simplex-Virus), CMV (Zytomegalievirus), Pneumocystis jiroveci und Aspergillus fumigatus sowie eine Zytologie der BAL-Flüssigkeit. Im 3. Schritt sollten Autoimmunerkrankungen mit Lungenbeteiligung wie ein systemischer Lupus erythematodes (SLE), eine Sklerodermie, eine granulomatöse Polyangiitis (GPA) oder eine

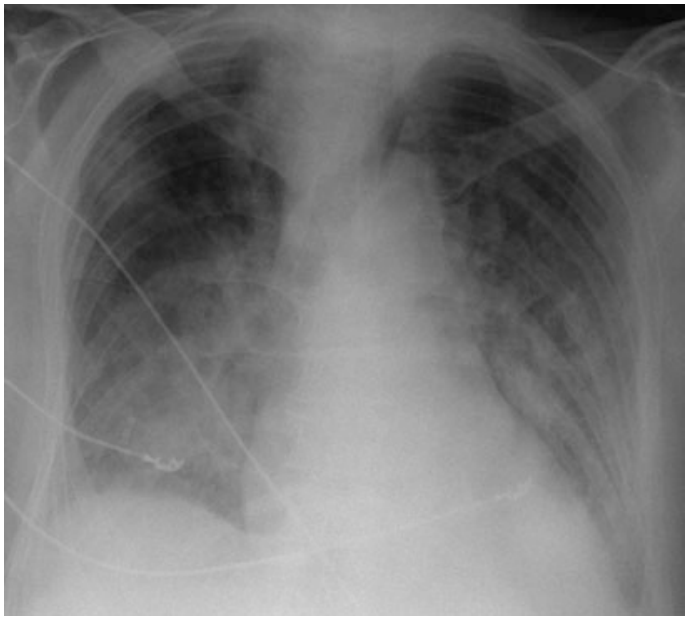

Abb. 1 Röntgen-Thorax bei ARDS: schwere nosokomiale Pneumonie

rheumatoide Arthritis ausgeschlossen werden. Eine Ultraschalluntersuchung von Lunge und Pleura, Abdomen, Herz und Gefäßen ermöglicht schnell wichtige Informationen über Vorliegen eines Pneumothorax, von Pleuraergüssen, Aussagen über die kardiale Funktion sowie über den Gefäßstatus vor Anlage eines extrakorporalen Verfahrens. Die in vielen Fällen ausreichende Röntgen-Thoraxaufnahme (Abb. 1) lässt sich bei spezieller Fragestellung (Pneumothorax, Verdacht auf pulmonale Aspergillose, Ausmaß der Lungenschädigung, unklarer abdomineller Fokus) durch eine Computertomografie (CT) ergänzen. Eine offene Lungenbiopsie ist in seltenen Einzelfällen sinnvoll, wenn alle anderen diagnostischen Möglichkeiten ausgeschöpft worden sind (Papazian et al. 2016).

Die Diagnose eines ARDS sollte nach 24-36 Stunden kritisch reevaluiert werden, da sich bis zu $17 \%$ der initial mit ARDS aufgenommenen Patienten unter invasiver Beatmung und optimiertem PEEP schnell bessern. Auf der anderen Seite wird oftmals die Diagnose ARDS nicht gestellt, insbesondere die milde Form wird in der Hälfte der Fälle übersehen, das schwere ARDS in $22 \%$ der Fälle (Bellani et al. 2016). 


\section{Differenzialdiagnostik}

Ein akutes hypoxämisches Atemversagen kann neben dem kardialen Lungenödem infolge von Linksherzversagen oder hochgradigen Klappenvitien auch durch eine diffuse alveoläre Hämorrhagie, eine interstitielle Pneumonie oder durch hämatoonkologische Erkrankungen wie Lymphome, Leukämien oder solide Tumoren verursacht werden. Des Weiteren sind pulmonale Folgeschäden einer nicht protektiven invasiven Beatmung (VILI, ,ventilator-induced lung injury“) zu erwägen. Weitere Differenzialdiagnosen sind eine akute Lungenarterienembolie, ein infektexazerbiertes Asthma bronchiale sowie eine Volumenüberladung im Rahmen eines akuten Nierenversagens oder in der Initialphase der Sepsistherapie.

\section{$7 \quad$ Therapie}

Grundlage jeder Therapie des ARDS-Patienten ist die Therapie der ursächlichen Grunderkrankung wie beispielsweise einer Pneumonie (siehe Kap. > „Untere Atemwegsinfektion/Ambulant erworbene Pneumonien“ und Kap.» „Nosokomiale Pneumonien").

Nichtinvasive oder invasive Beatmungstherapie, medikamentöse Therapie und supportive Maßnahmen haben das Ziel, atelektatische Lungenbereiche zu rekrutieren und damit das Shuntvolumen zu reduzieren, die Perfusion belüfteter Lungenareale $\mathrm{zu}$ erhöhen, das intraalveoläre/ interstitielle Ödem zu mobilisieren und zu einer Restitutio der alveolokapillären Einheit zu führen. Der Einsatz extrakorporaler Verfahren zur Lungenunterstützung kann bei Patienten mit schwerem ARDS und therapierefraktärer Hypoxämie im Einzelfall als Rescue-Verfahren erwogen werden, um protektive Beatmungsstrategien zu ermöglichen und um Zeit für die Ausheilung der Erkrankung zu gewinnen (,,bridging to recovery“).

\subsection{Nichtinvasive Beatmung (NIV) bei ARDS}

Bei Patienten mit mildem ARDS führt die NIV zu einer Entlastung der ventilatorischen Atemarbeit. Die Rekrutierung atelektatischer Lungenbereiche unter einem ausreichend hohen PEEP hat eine Verbesserung der Oxygenierung zur Folge. Der Einsatz von NIV erfordert eine gute Compliance des Patienten, eine intensive pflegerische Betreuung und Erfahrung mit der Maskenbeatmung. Das Risiko eines NIV-Versagens liegt bei mehr als $50 \%$ und korreliert mit der Schwere der Oxygenierungsstörung und der Erkrankungsschwere. Eine verzögerte Intubation kann die Mortalität erhöhen (Kluge 2016). Daher sollte bei Patienten mit schwerem ARDS $\left(\mathrm{PaO}_{2} / \mathrm{FiO}_{2} \leq 100 \mathrm{mmHg}\right)$ primär invasiv beatmet werden.

\subsection{High-Flow-Sauerstofftherapie (HFNC) bei ARDS}

Vergleichbare Ergebnisse liegen für den Einsatz der HFNC bei Patienten mit mildem ARDS vor. Dabei wird dem Patienten erwärmter und befeuchteter Sauerstoff mit einem Fluss von bis zu 60 1/min über spezielle nasale Kanülen zugeführt. Eine Reduktion der Atemarbeit, eine verbesserte Oxygenierung und $\mathrm{CO}_{2}$-Clearance und ein erhöhtes endexpiratorisches Lungenvolumen sowie eine leicht verminderte Mortalität gegenüber NIV wurden unter HFNC beschrieben.

\subsection{Invasive mechanische Beatmung (IMV) bei ARDS}

Grundlage der Beatmungseinstellung beim ARDS ist die lungenprotektive Beatmung mit Verwendung kleiner Tidalvolumina und einer Begrenzung des endinspiratorischen Atemwegsdrucks, um das Risiko einer beatmungsassoziierten Lungenschädigung zu minimieren, indem eine endinspi- 
ratorische Überdehnung von Alveolen vermieden wird, während ein adäquater positiver endexspiratorischer Druck (PEEP) einen endexpiratorisch Alveolarkollaps vermeiden soll. Die protektive invasive Beatmung orientiert sich dabei am Standardkörpergewicht des Patienten, da das Lungenvolumen durch die Körpergröße bestimmt ist und bei Adipositas nicht zunimmt.

Empfohlene Beatmungseinstellung bei ARDS (S3-Leitlinie Invasive Beatmung und Einsatz extrakorporaler Verfahren bei akuter respiratorischer Insuffizienz.):

- Tidalvolumen $\leq 6 \mathrm{ml} / \mathrm{kg}$ Standardkörpergewicht

- Standardkörpergewicht Männer $(\mathrm{kg})=50,0$ $+0,91 \times($ Größe in cm $-152,4)$

- Standardkörpergewicht Frauen $(\mathrm{kg})=45,5+$ $0,91 \times($ Größe in cm - 152,4)

- Endinspiratorischer Atemwegsdruck (Plateaudruck) $\leq 30 \mathrm{cmH}_{2} \mathrm{O}$

- Inspiratorische Druckdifferenz (,driving pressure"; Plateaudruck-PEEP) $\leq 15 \mathrm{cmH}_{2} \mathrm{O}$

- PEEP-Titrierung mit Orientierung am Oxygenierungsbedarf (Tab. 2)

- Sauerstoffsättigung 90-94\%

- Permissive Hyperkapnie (bis pH-Wert 7,2)

- Beatmung mit verlängerter Inspirationszeit (Inspiration:Exspiration 1:1-1:1,5) homogenere Ventilationsmöglichkeit reduziert sich das Risiko einer beatmungsinduzierten Lungenschädigung. Bauchlagerung wird für Patienten mit einem Oxygenierungsindex $\mathrm{PaO}_{2} / \mathrm{FiO}_{2}<$ $150 \mathrm{mmHg}$ früh im Krankheitsverlauf und für mindestens 16 Stunden/Tag empfohlen. Besonders wichtig sind die Sicherung des Beatmungszugangs und die Vermeidung von lagerungsbedingten Druckläsionen sowie ein geschultes Pflegeteam. Kontraindikationen sind Schwangerschaft, hämodynamische Instabilität, Hirndruck, offenes Abdomen sowie instabile Wirbelsäulenfrakturen.

\subsection{Rekrutierungsmanöver}

Eine kurzzeitige deutliche Erhöhung der Beatmungsdrücke (40-45 $\left.\mathrm{cmH}_{2} \mathrm{O}\right)$ kann zu einer alveolären Rekrutierung kollabierter Lungenbereiche führen. Insbesondere nach Bronchoskopie, Lagerung oder Diskonnektion von der Beatmung ist hierdurch kurzfristig eine verbesserte Oxygenierung zu erreichen. Bei ausgedehnten konsolidierten Lungenarealen ist dieser Effekt geringer. Rekrutierungsmanöver sind nicht standardisiert und unterscheiden sich hinsichtlich der Spitzendrücke, der Dauer und Häufigkeit ihrer Anwendung. Der routinemäßige Einsatz von Rekrutierungsmanövern wird momentan, auch aufgrund des Risikos von Baro- und Volutraumen, nicht empfohlen.

\subsection{Medikamentöse Therapie}

Insbesondere in der Frühphase des moderaten bis schweren ARDS führt die Bauchlagerung durch eine veränderte Einwirkung der Schwerkraft zu einer Rekrutierung der dorsalen Lungenabschnitte. Dies bewirkt ein verbessertes Ventilations-Perfusions-Verhältnis mit einer erhöhten $\mathrm{CO}_{2}$-Clearance und $\mathrm{O}_{2}$-Aufnahme. Durch eine

Tab. 2 Voraussichtlicher PEEP in Abhängigkeit vom Sauerstoffbedarf $\left(\mathrm{FiO}_{2}\right)$

\begin{tabular}{|l|l|l|l|l|l|l|l|l|} 
FiO $_{2}$ & 0,3 & 0,4 & 0,5 & 0,6 & 0,7 & 0,8 & 0,9 & 1,0 \\
\hline PEEP & 5 & $5-8$ & $8-10$ & 10 & $10-14$ & 14 & $14-18$ & $18-24$ \\
\hline
\end{tabular}

Neben der Therapie der Grunderkrankung, wie einer antiinfektiven Therapie bei Pneumonie oder Sepsis, ist in Abhängigkeit von Hämodynamik und Gewebeperfusion grundsätzlich beim ARDS-Patienten ein restriktives Flüssigkeitsregime anzustreben. Hierunter lässt sich eine verbesserte Oxygenierung sowie eine verkürzte invasive Beatmungsdauer und Intensivliegedauer nachweisen.

Bei schwerer therapierefraktärer Hypoxämie kann der Einsatz von inhalativem Stickstoffmonoxid (iNO) erwogen werden. iNO bewirkt 
eine Vasodilatation und kann hierdurch $\mathrm{zu}$ einer verbesserten Oxygenierung durch Reduktion der intrapulmonalen Shunts führen. Insbesondere bei Rechtsherzbelastung ist die Widerstandsabnahme der pulmonal-arteriellen Gefäßstrombahn im Sinne einer rechtskardialen Nachlastsenkung vorteilhaft. Unter iNO wird jedoch eine erhöhte Inzidenz von Nierenfunktionsstörungen beschrieben, sodass kein routinemäßiger Einsatz bei Patienten mit ARDS erfolgen sollte. Inhalative Prostaglandine wirken ebenfalls selektiv pulmonal-arteriell vasodilatierend, werden aktuell aber ebenfalls nicht für den Routineeinsatz bei ARDS-Patienten empfohlen.

Für den Einsatz von Muskelrelaxanzien in der Frühphase des ARDS $\left(\mathrm{PaO}_{2} / \mathrm{FiO}_{2}<150 \mathrm{mmHg}\right)$ liegen prospektive Studiendaten für Cisatracurium vor. Neben einer verringerten 90-Tage-Sterblichkeit bei Patienten mit $\mathrm{PaO}_{2} / \mathrm{FiO}_{2}<120 \mathrm{mmHg}$ wurden mehr beatmungsfreie Tage, weniger Tage mit Organversagen und Pneumothoraces (vermindertes Bio- und Barotrauma) bei vergleichbarer Zahl an Critical-Illness-Myopathien gefunden (Papazian et al. 2010). Durch das erhöhte Risiko einer beatmungsinduzierten Zwerchfelldysfunktion (VIDD, ,ventilator-induced diaphragmale dysfunction") und die noch offene Studienlage wird der Einsatz von Muskelrelaxanzien aktuell nicht routinemäßig empfohlen.

Eine zusätzliche Therapie mit Kortikosteroiden hat unabhängig von einer bakteriellen oder viralen Infektion weder in der inflammatorischen Frühphase noch in der proliferativen und fibrosierenden Spätphase des ARDS zu einer verbesserten Prognose geführt. Ausgenommen sind ARDSPatienten, die im Rahmen einer pulmonalen Manifestation einer zugrunde liegenden Autoimmunerkrankung eine hoch dosierte Steroidtherapie erhalten. Unter Kortikosteroiden ist das potenzielle Risiko einer bakteriellen Sekundärinfektion erhöht und die Entwicklung einer steroidinduzierten Polyneuromyopathie zu berücksichtigen.

\subsection{Extrakorporale Lungenersatzverfahren}

In den letzten Jahren wurden, auch bedingt durch technologische Innovationen der verwendeten Systeme mit Verbesserung von Oxygenatoren, Pumpen und Kanülen, zunehmend extrakorporale Verfahren in der Therapie des schweren pulmonalen Organversagens angewendet. Prinzipiell lassen sich hierbei Systeme, die primär der Oxygenierung dienen (extrakorporale Membranoxygenierung, ECMO), von Systemen zur extrakorporalen $\mathrm{CO}_{2}$-Eliminierung unterscheiden. Klassische Indikation für eine ECMO-Therapie ist das schwerste Lungenversagen, bei dem eine adäquate Oxygenierung mit konventionellen Maßnahmen nicht mehr möglich ist. Allerdings sind Aufwand und potenzielle Komplikationen dieser Therapieform nicht unerheblich (Kluge et al. 2017).

Durch den Einsatz von Systemen zur extrakorporalen $\mathrm{CO}_{2}$-Eliminierung kann bei moderater Einschränkung der Oxygenierung und ausgeprägter Hyperkapnie eine effektive $\mathrm{CO}_{2}$-Elimination erreicht werden. Diese Systeme werden daher bei ARDS eingesetzt, um die Etablierung einer protektiven Beatmung zu ermöglichen. Allerdings fehlen bislang Studien, die einen Nutzen der so behandelten Patienten nachweisen können. Die S3-Leitlinie „Invasive Beatmung und Einsatz extrakorporaler Verfahren bei akuter respiratorischer Insuffizienz" nimmt ausführlich Stellung zu Indikationen und Kontraindikationen der extrakorporalen Verfahren zur Lungenunterstützung (S3-Leitlinie Invasive Beatmung und Einsatz extrakorporaler Verfahren bei akuter respiratorischer Insuffizienz. 2017).

Beratung und Unterstützung durch das nächstgelegene ARDS-Zentrum sollte frühzeitig eingeholt werden. Eine 24-Stunden-Erreichbarkeit ermöglicht bei instabilen Patienten eine ambulante ECMO-Anlage mit sicherem Transport in das nächste ARDS-Zentrum. 
8 Verlauf und Prognose

Patienten mit ARDS haben auch heute noch ein hohes Risiko, an ihrer Erkrankung zu versterben. Neben der erfolgreichen Therapie der Grunderkrankung können die Implementierung einer protektiven Beatmung mit Tidalvolumina $\leq 6 \mathrm{ml} / \mathrm{kg}$ Standardkörpergewicht, der adäquat adjustierte PEEP und die frühe Bauchlagerung bei schwererem ARDS zu einer reduzierten Mortalität beitragen. Daten der Lung-Safe-Studie zeigen jedoch, dass zwei Drittel der Patienten mit einem Tidalvolumen $\geq 8 \mathrm{ml} / \mathrm{kg}$ Standardgewicht und $83 \%$ der Patienten mit einem PEEP $<12 \mathrm{cmH}_{2} \mathrm{O}$ beatmet wurden und die Bauchlage nur bei $16 \%$ der Patienten mit schwerem ARDS durchgeführt wurde.

Die Krankenhausmortalität liegt für Patienten mit mildem ARDS bei $35 \%$ und steigt bei moderatem ARDS auf $40 \%$, bei schwerem ARDS auf $46 \%$ an (Bellani et al. 2016). Diese Daten entsprechen ungefähr der retrospektiven Auswertung der klinischen Datenbanken der Berlin-Definition 2011. Eine hohe Mortalität von $66 \%$ weisen ARDS-Patienten ohne klassische Risikofaktoren auf (Gibelin et al. 2016).

Patienten mit moderatem bis schwerem ARDS haben häufig einen prolongierten WeaningVerlauf, der oftmals zu einer Verlegung in ein spezialisiertes Weaning-Zentrum führt. Der frühe Einsatz von Spontanatmung, protokollbasierte Sedierungspausen, intensive Physiotherapie mit einer frühen Mobilisation, ein aufmerksames Delirmanagement und eine bedarfsadaptierte Ernährung wirken sich später entscheidend auf den Weaning-Erfolg aus.

Zunehmend befassen sich Studien mit den Langzeitfolgen der Erkrankung. Neben einer oftmals weitgehend wiederhergestellten pulmonalen Funktion ist die körperliche Leistungsfähigkeit auch 3 Jahre nach der Erkrankung deutlich eingeschränkt. Valider Parameter ist die 6-MinutenGehstrecke, die zu diesem Zeitpunkt noch um $33 \%$ reduziert ist. Nach 2 Jahren leiden $46 \%$ der Patienten unter neurokognitiven Störungen, die das Gedächtnis, die Aufmerksamkeit und die Konzentrationsfähigkeit betreffen. Bei $40 \%$ der Patienten liegt zu diesem Zeitpunkt eine depres- sive Symptomatik vor. Eine posttraumatische Belastungsstörung betrifft Patienten wie Angehörige in gleichem Maße. Die Hälfte der vorher im Arbeitsleben stehenden Patienten ist $1 \mathrm{Jahr}$ nach der Erkrankung wieder berufstätig, nach 5 Jahren sind es $77 \%$ der Patienten (Dodoo-Schnittke et al. 2017). Es wird deutlich, dass viele Patienten über die Rehabilitationsphase hinaus das Angebot einer langfristigen medizinischen Anbindung brauchen, um die Langzeitfolgen der ARDS-Erkrankung in einem veränderten Alltagskontext bewältigen zu können.

\section{Literatur}

Bellani G, Laffey JG, Pham T et al (2016) Epidemiology, patterns of care, and mortality for patients with acute respiratory distress syndrome in intensive care units in 50 countries. JAMA 315(8):788-800

Deutsche Gesellschaft für Pneumologie und Beatmungsmedizin (DGP) (2015) S3-Leitlinie: Nichtinvasive Beatmung als Therapie der akuten respiratorischen Insuffizienz. AWMF Leitlinien-Register Nr. 020-004, Stand: 10.07.2015. https://www.awmf.org/leitlinien/ detail/11/020-004.html. Zugegriffen am 19.11.2018

Deutsche Interdisziplinäre Vereinigung für Intensiv- und Notfallmedizin (DIVI) et al. (2017) S3-Leitlinie: Invasive Beatmung und Einsatz extrakorporaler Verfahren bei akuter respiratorischer Insuffizienz. AWMF Leitlinien-Register Nr. 001/021, Stand 04.12.2017. https:/www.awmf.org/uploads/tx_szleitlinien/001-0211_ S3_Invasive_Beatmung_2017-12.pdf. Zugegriffen am 19.11.2018

Dodoo-Schnittke F, Brandstetter S, Blecha S et al (2017) Determinants of quality of life and return to work following acute respiratory distress syndrome-a systematic review. Dtsch Arztebl Int 114:103-109

Gibelin A, Parrot A, Maitre B et al (2016) Acute respiratory distress syndrome mimickers lacking common risc factors of the Berlin Definition. Intensive Care Med 42:164-172

Kluge S (2016) ARDS - Extrakorporaler Lungenersatz, Airway-Management. In: Welte T, Kluge S, Janssens U, Tacke F (Hrsg) Handbuch Intensivmedizin 2017, Bd 1. med publico GmbH, Wiesbaden, S 1-39

Kluge S, Sensen B, Braune S (2017) Extrakorporale Lungenunterstützung bei Patienten mit ARDS - extracorporeal lung support in patients with ARDS. Dtsch Med Wochenschr 142:88-95

Papazian L, Forel JM, Gacouin A et al (2010) Neuromuscular blockers in early acute respiratory distress syndrome. N Engl J Med 363:1107-1116

Papazian L, Calfee CS, Chiumello D et al (2016) Diagnostic workup for ARDS patients. Intensive Care Med 42:674-685 
Ranieri VM, Rubenfeld GD, Thompson ET et al (2012) Acute respiratory distress syndrome: the Berlin Definition. JAMA 307(23):2526-2533

Umbrello M, Formenti P, Bolgiaghi L et al (2017) Current concepts of ARDS: a narrative review. Int J Mol Sci 18:64. https://doi.org/10.3390/ijms18010064

\section{Internet}

www.ardsnet.org: Homepage des NIH-NHLBI ARDSNetzwerkes USA

www.ardsnetwork.de: Homepage des ARDS-Netzwerkes Deutschland

www.awmf.org: Homepage der Arbeitsgemeinschaft der Wissenschaftlichen Medizinischen Fachgesellschaften e. V.

www.elso.org: Homepage der Extracorporeal Life Support Organisation (ELSO) 